\title{
Homoclinic and period-doubling bifurcations for damped systems
}

\author{
by
}

Ugo BESSI

Scuola Normale Superiore, Piazza Cavalieri 7, 56100 Pisa, Italy

ABSTRACT. - We consider the following problem

$$
\left\{\begin{array}{c}
\ddot{u}=-V^{\prime}(u, t)-\alpha \dot{u} \\
u(-\infty)=\dot{u}(-\infty)=0=u(\infty)=\dot{u}(\infty)
\end{array}\right.
$$

where $V \in C^{2}\left(\mathbf{R}^{n} \times \mathbf{R}, \mathbf{R}\right)$ is one-periodic in time and behaves roughly like $V(x, t) \simeq-\frac{1}{2}|x|^{2}+|x|^{\mu}$ for some $\mu>2$. It has been shown in [6] that $0 \in \mathbf{R}^{2 n}$ is a hyperbolic fixed point for $\Phi_{\alpha}$, the step-1 map associated to equation $\left(P^{\alpha}\right)$, and that $\Phi_{0}$ has points homoclinic to 0, i.e. $\left(P^{0}\right)$ has a solution not identically 0 . We will show that, if the solutions of $\left(P^{0}\right)$ are isolated in $H^{1}\left(\mathbf{R}, \mathbf{R}^{n}\right)$, then $\Phi_{\alpha}$ has positive topological entropy for $\alpha \in[0, \bar{\alpha}]$ for some $\bar{\alpha}>0$. Moreover, we will follow a branch of solutions of $\left(P^{\alpha}\right)$ as $\alpha$ varies in $\mathbf{R}^{+}$up to an homoclinic tangency and show the existence of an infinite cascade of period-doubling bifurcations near this tangency.

Key words: Homoclinic bifurcations, variational methods.

RÉSUMÉ. - Nous considérons le problème suivant

$$
\left\{\begin{array}{c}
\ddot{u}=-V^{\prime}(u, t)-\alpha \dot{u} \\
u(-\infty)=\dot{u}(-\infty)=0=u(\infty)=\dot{u}(\infty)
\end{array}\right.
$$

où $V \in C^{2}\left(\mathbf{R}^{n} \times \mathbf{R}, \mathbf{R}\right)$ est périodique en temps de période 1 et se comporte comme $V(x, t)=\simeq-\frac{1}{2}|x|^{2}+|x|^{\mu}$, avec $\mu>2$. Il a été

Classification A.M.S.: 34 C 35,34 C 28,34 C 23.

Annales de l'Institut Henri Poincaré - Analyse non linéaire - 0294-1449 
montré dans [6] que $0 \in \mathbf{R}^{2 n}$ est un point fixe hyperbolique pour $\Phi_{\alpha}$, l'application de temps 1 associée à l'équation $\left(P^{\alpha}\right)$, et que $\Phi_{0}$ a des points homoclines à 0 , i.e. que $P^{0}$ a une solution non identiquement nulle. Nous prouvons que, si les solutions de $\left(P^{0}\right)$ sont isolées dans $H^{1}\left(\mathbf{R}, \mathbf{R}^{n}\right)$, alors $\Phi_{0}$ a une entropie topologique positive si $\alpha \in[0, \bar{\alpha}]$ avec $\bar{\alpha}>0$. De plus, nous suivons une branche de solutions de $\left(P^{\alpha}\right)$ quand $\alpha$ varie dans $\mathbf{R}^{+}$ jusqu'à une tangente homocline et nous prouvons l'existence d'une cascade infinie de bifurcations avec doublement de périodes près de cette tangente.

\section{INTRODUCTION}

In [3] the following problem was considered

$$
\left\{\begin{array}{c}
\ddot{u}=-V^{\prime}(u, t)-\alpha \dot{u} \\
u(-\infty)=\dot{u}(-\infty)=0=u(\infty)=\dot{u}(\infty)
\end{array}\right.
$$

where the potential $V(x, t) \in C^{3}\left(\mathbf{R}^{p} \times \mathbf{R}, \mathbf{R}\right)$ is one-periodic in time and behaves roughly like

$$
V(x, t)=-\frac{1}{2}|x|^{2}+W(x, t), \quad W(x, t) \simeq|x|^{\mu}, \quad \mu>2 .
$$

It was proven that, if $V$ is "really" time-dependent in a sense to be specified in Section 1, then problem $\left(P^{\alpha}\right)$ has, as $\alpha$ varies in $\mathbf{R}^{+}$, a global branch of solutions containing a nontrivial solution $\bar{u}$ of $\left(P^{0}\right)$. Such a branch is not reduced to the only $\bar{u}$ and has a turning point whenever it is bounded. The author also exhibited situations in which all continua of solutions of $\left(P^{\alpha}\right)$ are bounded. The present paper continues the investigations of [3] and gives a more precise account of the bifurcations of $\left(P^{\alpha}\right)$.

Let us consider the step-1 map $\Phi_{\alpha}$ associated to $\left(P^{\alpha}\right)$, i.e. the map

$$
\Phi_{\alpha}: \quad(x, y) \rightarrow(u(1), \dot{u}(1))
$$

where $u$ solves

$$
\left\{\begin{array}{c}
\ddot{u}=-V^{\prime}(u, t)-\alpha \dot{u} \\
u(0)=x \\
\dot{u}(0)=y .
\end{array}\right.
$$

In [14] it has been shown that it is possible to embed a Bernoulli shift in the dynamics of $\Phi_{0}$; we show in theorem 1.1 that, slightly strenghtening 
the hypotheses of [14], this shift can be continued to a positive $\bar{\alpha}$. Since our hypotheses do not imply hyperbolicity, this fact does not follow at once from the general theory of dynamical systems. Analogously as in [14], the presence of a Bernoulli shift has as a corollary that the step-1 map of our equation has positive topological entropy for $\alpha \in[0, \bar{\alpha}]$. This corollary does not follow directly from [14] since in general situations the topological entropy of a map is not expected to be a lower semi-continuous function of the map (see for instance [11]).

The turning point of the branch of homoclines can be seen as a point of non-transversal intersection for the stable and unstable manifolds of $\Phi_{\alpha}$. Since the unfolding of an homoclinic tangency usually exhibits (see for instance [1] and its generalization [7]) an infinite cascade of period-doubling bifurcations, we tried to see if such is the case for our equation. We show in theorem 2.1 that a cascade of period-doubling bifurcations actually occurs. We remark that, for $p$ even, such a result is not covered by [7].

The methods we use are very simple; as in [6] and in [3], we consider the homoclinics as zeroes of an operator defined on $H^{1}\left(\mathbf{R}, \mathbf{R}^{p}\right)$, whose study we simplify through the finite-dimensional reduction of Morse. Once this reduction is done, we rely on the properties of the topological degree to get our result.

\section{SECTION 1}

In the course of this paper, $\phi_{R}$ will denote a $C^{\infty}$ real function such that $\phi_{R}(x)=1$ for $x \in[-R, R], \phi_{R}(x)=0$ for $x \notin[-R-1, R+1]$ and $\left\|\dot{\phi}_{R}\right\|_{\infty} \leq 2$. By $B_{p}(x, r), B_{2 p}(x, r)$ we will denote the open balls of $\mathbf{R}^{p}$ and $\mathbf{R}^{2 p}$ respectively, and by $\langle\cdot, \cdot\rangle$ the internal product. By $[x]$ we will denote the greatest integer smaller or equal than $x$.

We consider a potential of the form

$$
V(x, t)=-\frac{1}{2}|x|^{2}+W(x, t)
$$

and make the following assumptions on $W$.

$$
\begin{gathered}
W 1) \quad W \in C^{3}\left(\mathbf{R}^{p} \times \mathbf{R}, \mathbf{R}\right) \quad \text { and } W \text { is } 1 \text {-periodic in time } \\
W^{\prime \prime}(0, t)=0, \quad \forall t \in \mathbf{R}, \\
W 2) \quad\left\{\begin{array}{c}
\exists \mu>2: \quad \begin{array}{c}
0 \leq \mu W(x, t) \leq\left\langle x, W^{\prime}(x, t)\right\rangle, \\
\forall(x, t) \in \mathbf{R}^{p} \times \mathbf{R}
\end{array}
\end{array}\right.
\end{gathered}
$$


W4) $\left\langle x, W^{\prime}(x, t)\right\rangle<W^{\prime \prime}(x, t)(x, x), \quad \forall(x, t) \in\left(\mathbf{R}^{p} \backslash\{0\}\right) \times \mathbf{R}$

W5) there is an open set $D \subset \mathbf{R}^{p}$ such that

$$
0 \in D, \quad\left\langle-V^{\prime}(x, t), x\right\rangle>0, \quad \forall x \in D \quad \text { and }
$$

$\exists b<0$ such that $\partial D \subset\{V<b\}, \quad V$ is autonomous in $\{V<b\}$

The following two hypotheses are to insure that $V$ is "really" timedependent (see for instance [14], [6], [3]). We remark that, if $W(x, t)=$ $W_{1}(x)+\varepsilon W_{2}(x, t)$ with $\varepsilon$ small, they are closely related to the classical Melnikov condition (see for instance [4], [2], [9]). We will assume the following.

$$
\text { The solutions of }\left(P^{0}\right) \text { are isolated in } H^{1}\left(\mathbf{R}, \mathbf{R}^{p}\right)
$$

( $\star) \quad \forall \alpha \geqq 0$, the solutions of $\left(P^{\alpha}\right)$ are at most countable.

We recall from [6] that $\left(P^{0}\right)$ is a variational problem and that its solutions are the critical points of the functional

$$
f: \quad H^{1}\left(\mathbf{R}, \mathbf{R}^{p}\right) \rightarrow \mathbf{R}, \quad f: \quad u \rightarrow \int_{R}\left[\frac{1}{2}|\dot{u}|^{2}-V(u, t)\right] d t
$$

In [6] it has been shown that, if $W 1-3$ ) hold, then $f$ has a non-trivial Mountain Pass critical point $\bar{u}$. In [3], lemma 1.4, it has been shown that, if $W 5$ ) holds, there is $\alpha_{0}>0$ such that for $\alpha \geq \alpha_{0},\left(P^{\alpha}\right)$ has no solutions. We also recall that, by lemma 1.2 of [3], there is $\bar{a}>0$ such that any $u$ which solves $\left(P^{\alpha}\right)$ for any $\alpha \geq 0$ satisfies $\|u\|_{\infty}>\bar{a}$.

We now state a consequence of the stable and unstable manifold theorem (see for instance [12]) that we will need later on. By this theorem, we can take $a \in(0, \bar{a})$ so small that, for $\alpha \in\left[0, \alpha_{0}\right]$, in $B_{p}(0, a) \times B_{p}(0, a)$ the local stable manifold of $\Phi_{\alpha}$ is the graph of a map from the stable eigenspace at 0 to the unstable one, and this map has zero derivative at 0 . From the implicit function theorem now follows that, possibly for a smaller $a$, the local stable manifold at 0 is the graph of a map from configuration space to velocity space. With the same argument, also the local unstable manifold in $B_{p}(0, a) \times B_{p}(0, a)$ is the graph of a map from configuration space to velocity space. For such an $a$ we define

$$
\underline{t}=\sup \left\{n \in \mathbf{Z}: \bar{u}((-\infty, n]) \subset B_{p}\left(0, \frac{a}{2}\right)\right\}
$$




$$
\bar{t}=\inf \left\{n \in \mathbf{Z}: \bar{u}([n, \infty)) \subset B_{p}\left(0, \frac{a}{2}\right)\right\} .
$$

The previous considerations imply that, for any $x \in B_{p}(0, a), \alpha \in\left[0, \alpha_{0}\right]$ and $j \in \mathbf{Z}$, there is $v_{-\infty}^{\text {arr }}(x, \alpha)$ depending at least $C^{3}$ on its parameters such that the solution of

$$
\left\{\begin{array}{c}
\ddot{u}=-V^{\prime}(u, t)-\alpha \dot{u} \\
u(j)=x \\
\dot{u}(j)=v_{-\infty}^{\text {arr }}(x, \alpha)
\end{array} \quad P(j, x, \alpha,-\infty)\right.
$$

satisfies $u(t) \rightarrow 0$ for $t \rightarrow-\infty$. Analogously, for $y \in B_{p}(0, a)$, $\alpha \in\left[0, \alpha_{0}\right]$ and $j \in \mathbf{Z}$, there is a $C^{3}$ function $v_{\infty}^{\text {dep }}(y, \alpha)$ such that the solution of

$$
\left\{\begin{array}{l}
\ddot{u}=-V^{\prime}(u, t)-\alpha \dot{u} \\
u(j)=y \\
\dot{u}(j)=v_{\infty}^{\operatorname{dep}}(y, \alpha)
\end{array} \quad P(j, y, \alpha, \infty)\right.
$$

satisfies $u(t) \rightarrow 0$ for $t \rightarrow \infty$.

We recall that in [6] the following minimax class was introduced

$$
\Delta=\left\{g \in C\left([0,1], H^{1}\left(\mathbf{R}, \mathbf{R}^{p}\right)\right): g(0)=0, g(1) \in\{f<0\}\right\}
$$

and that in [3] it has been shown that, under hypotheses $W 1-4$ ), there is critical point $\vec{u}$ of $f$ such that

$$
f(\bar{u})=\inf _{g \in \Delta} \max _{s \in[0,1]} f(g(s)) .
$$

We recall that $g$ is usually called a mountain pass path, and $\bar{u}$ a mountain pass critical point. We are now going to prove the following.

THEOREM 1.1. - Let W1-4) and ( $\star$ ) hold. Then $\forall \varepsilon>0$ there is $\bar{\alpha}>0$ and $N \in \mathbf{N}$ such that for any sequence $\left\{m_{j}\right\}_{j \in \mathrm{Z}}$ satisfying $m_{j}-m_{j-1} \geq 2 \mathrm{~N}$, there is a continuum $\Theta_{\left\{m_{j}\right\}_{j \in Z}} \subset H_{\mathrm{loc}}^{1}\left(\mathbf{R}, \mathbf{R}^{p}\right) \times[0, \bar{\alpha}]$ of solutions of

such that

$$
\ddot{u}=-V^{\prime}(u, t)-\alpha \dot{u}
$$

$$
\begin{aligned}
& \Theta_{\left\{m_{j}\right\}_{j \in Z}} \cap H_{\mathrm{loc}}^{1}\left(\mathbf{R}, \mathbf{R}^{p}\right) \times\{0\} \neq \varnothing, \\
& \Theta_{\left\{m_{j}\right\}_{j \in Z}} \cap H_{\mathrm{loc}}^{1}\left(\mathbf{R}, \mathbf{R}^{p}\right) \times\{\bar{\alpha}\} \neq \varnothing
\end{aligned}
$$

and $\forall(u, \alpha) \in \Theta_{\left\{m_{j}\right\}_{j \in Z}}$ we have

$$
\forall j \in \mathbf{Z}, \quad\left\|u\left(\cdot+m_{j}\right) \phi_{N}-\bar{u}\right\|_{H^{1}}\left(\mathbf{R}, \mathbf{R}^{p}\right) \leq \varepsilon .
$$

Vol. $12, n^{\circ} 1-1995$. 
Moreover, if the sequence $\left\{m_{j}\right\}_{j \in \mathbf{Z}}$ is finite, then every $(u, \alpha) \in \Theta_{\left\{m_{j}\right\}_{j \in Z}}$ solves $\left(P^{\alpha}\right)$, while, if $\left\{m_{j}\right\}_{j \in \mathbf{Z}}$ is periodic of period l, then the elements of $\Theta_{\left\{m_{j}\right\}_{j \in Z}}$ are periodic of period $m_{l}-m_{0}$.

Proof. - The proof is divided into 4 steps.

Step 1. - This is the classical finite-dimensional reduction of Morse. We consider a partition $t_{1}<t_{2}<\ldots<t_{k}$ of $\mathbf{R}$ with $t_{1}, t_{k} \in \mathbf{Z}, t_{1} \leq \underline{t}, t_{k} \geq \bar{t}$ and such that there are no Jacobi fields between $\bar{u}\left(t_{i}\right)$ and $\bar{u}\left(t_{i+1}\right)$. For such a partition $t_{1}, \ldots, t_{k}$, the implicit function theorem implies that there is $\delta \in\left(0, \frac{a}{2}\right)$ so small that, for any $i \in(1, \ldots, k-1), \alpha \in[0, \delta]$ and for any $(x, y) \in B_{p}\left(\bar{u}\left(t_{i}\right), \delta\right) \times B_{p}\left(\bar{u}\left(t_{i+1}\right), \delta\right)$, there are $v^{\mathrm{dep}}(x, y, \alpha, i)$, $v^{\operatorname{arr}}(x, y, \alpha, i+1)$ depending at least $C^{3}$ on their variables such that the solution of

$$
\left\{\begin{array}{c}
\ddot{u}=-V^{\prime}(u, t)-\alpha \dot{u} \\
u\left(t_{i}\right)=x \\
\dot{u}\left(t_{i}\right)=v^{\operatorname{dep}}(x, y, \alpha, i)
\end{array} \quad P(x, y, \alpha, i)\right.
$$

satisfies $u\left(t_{i+1}\right)=y$ and $\dot{u}\left(t_{i+1}\right)=v^{\mathrm{arr}}(x, y, \alpha, i+1)$. Moreover, for $\alpha \in[0, \delta]$ we have the immersion

$$
\begin{gathered}
i_{\alpha}: \quad \prod_{i=1}^{k} B_{p}\left(\bar{u}\left(t_{i}\right), \delta\right) \subset \mathbf{R}^{k p} \rightarrow H^{1}\left(\mathbf{R}, \mathbf{R}^{p}\right) \\
i_{\alpha}:\left(x_{1}, \ldots, x_{k}\right) \rightarrow u(t)=\left\{\begin{array}{c}
u_{0}(t), \quad t \leq t_{1} \\
u_{i}(t), \quad \begin{array}{c}
t_{i} \leq t \leq t_{i+1}, \quad 1 \leq i \leq k-1 \\
u_{k}(t), \quad t_{k} \leq t
\end{array}
\end{array}\right.
\end{gathered}
$$

where $u_{0}$ solves $P\left(t_{1}, x_{1}, \alpha,-\infty\right), u_{i}$ solves $P\left(x_{i}, x_{i+1}, \alpha, i\right)$ for $1 \leq i \leq k-1$ and $u_{k}$ solves $P\left(t_{k}, x_{k}, \alpha, \infty\right)$. It is easy to check that $i_{\alpha}$ is continuous jointly in $\left(x_{1}, \ldots, x_{k}\right)$ and $\alpha$.

Given $\mathbf{y}=\left(y_{1}, \ldots, y_{k}\right) \in \mathbf{R}^{k p}$, we set

$$
h^{\mathbf{y}}(t)=\left\{\begin{array}{c}
h_{0}(t), \quad t \leq t_{1} \\
h_{i}(t), \quad t_{i} \leq t \leq t_{i+1}, \quad 1 \leq i \leq k-1 \\
h_{k}(t), \quad t_{k} \leq t
\end{array}\right.
$$

where $h_{0}$ solves

$$
\left\{\begin{array}{c}
\ddot{h}=-V^{\prime \prime}(\bar{u}, t) h \\
h\left(t_{1}\right)=y_{1} \\
h(-\infty)=0
\end{array}\right.
$$


$h_{i}, 1 \leq i \leq k-1$, solves

$$
\left\{\begin{array}{c}
\ddot{h}=-V^{\prime \prime}(\bar{u}, t) h \\
h\left(t_{i}\right)=y_{i} \\
h\left(t_{i+1}\right)=y_{i+1}
\end{array} \quad Q\left(i, y_{i}, y_{i+1}\right)\right.
$$

and $h_{k}$ solves

$$
\left\{\begin{array}{c}
\ddot{h}=-V^{\prime \prime}(\bar{u}, t) h \\
h\left(t_{k}\right)=y_{k} \\
h(\infty)=0 .
\end{array} \quad Q\left(k, y_{k}\right)\right.
$$

The solutions of the first and the last problems exist since in $B_{p}(0, a) \times B_{p}(0, a)$ the tangent spaces to the local stable and unstable manifolds of $\Phi_{0}$ project surjectively on the configuration space. The solution to the second problem exists since we choose our partition $t_{1}<\ldots<t_{k}$ in such a way, that there are no Jacobi fields between $\bar{u}\left(t_{i}\right)$ and $\bar{u}\left(t_{i+1}\right)$. We have clearly that

$$
i_{0}^{\prime}\left(\bar{u}\left(t_{1}\right), \ldots, \bar{u}\left(t_{k}\right)\right) \mathbf{y}=h^{\mathbf{y}} .
$$

We are now going to show that, given $\gamma>0$, we can find a partition of $\mathbf{R} t_{1}<\ldots<t_{k}$ with $t_{1}, t_{k} \in \mathbf{Z}$ and $t_{1} \leq \underline{t}, t_{k} \geq \bar{t}$, so fine that there are no Jacobi fields between $\bar{u}\left(t_{i}\right)$ and $\bar{u}\left(t_{i+1}\right)$ and that it also satisfies

$$
\left\|i_{0}^{\prime}\left(\bar{u}\left(t_{1}\right), \ldots, \bar{u}\left(t_{k}\right)\right)\left(\bar{u}\left(t_{1}\right), \ldots, \bar{u}\left(t_{k}\right)\right)-\bar{u}\right\|_{H^{1}}\left(\mathbf{R}, \mathbf{R}^{p}\right) \leq \gamma
$$

Indeed, it is easy to see that there is an integer $t_{1} \leq \underline{t}$ such that, for $h$ solving $Q\left(0, \bar{u}\left(t_{1}\right)\right)$, we have

$$
\int_{-\infty}^{t_{1}}\left[|\dot{h}|^{2}+|\dot{\bar{u}}|^{2}+|h|^{2}+|\bar{u}|^{2}\right] d t \leq \frac{\gamma^{2}}{3} .
$$

Analogously, for $T \geq \bar{t}$ integer and big enough and $h$ solving $Q(T, \bar{u}(T))$, we have

$$
\int_{T}^{\infty}\left[|\dot{h}|^{2}+|\dot{\bar{u}}|^{2}+|h|^{2}+|\bar{u}|^{2}\right] d t \leq \frac{\gamma^{2}}{3} .
$$

Once $t_{1}, T$ are thus chosen, it is easy to see that, given $\bar{\gamma}>0$, there is a sufficiently fine partition $t_{1}<\ldots<t_{k}=T$ of $\left[t_{1}, T\right]$, such that any $h_{i}$ solving $Q\left(i, \bar{u}\left(t_{i}\right), \bar{u}\left(t_{i+1}\right)\right)$ satisfies

$$
\sup _{\left[t_{i}, t_{i+1}\right]}\left[\left|\dot{h}_{i}(t)-\dot{\bar{u}}(t)\right|+\left|h_{i}(t)-\bar{u}(t)\right|\right] \leq \bar{\gamma}
$$


If in the last formula we choose $\bar{\gamma} \leq \frac{\gamma}{\sqrt{3\left(T-t_{1}\right)}}$ we have that

$$
\begin{aligned}
\mathbf{y} & =\left(\bar{u}\left(t_{1}\right), \ldots, \bar{u}\left(t_{k}\right)\right) \\
& \Rightarrow \int_{t_{1}}^{t_{k}}\left[\left|\dot{h}^{\mathbf{y}}(t)-\dot{\bar{u}}(t)\right|^{2}+\left|h^{\mathbf{y}}(t)-\bar{u}(t)\right|^{2}\right] d t \leq \frac{\gamma^{2}}{3} .
\end{aligned}
$$

Formula (1.3) is now implied by (1.4), (1.5) and (1.6).

Since in [3] we have shown that, if $W 4$ ) holds, the functional defined in (1.1) satisfies

$$
f^{\prime \prime}(\bar{u})(\bar{u}, \bar{u})=\int_{\mathbf{R}}\left[W^{\prime}(\bar{u}, t) \bar{u}-W^{\prime \prime}(\bar{u}, t)(\bar{u}, \bar{u})\right] d t<0
$$

formula (1.3) and the continuity of $f^{\prime \prime}$ imply that we can choose our partition $t_{1}, \ldots, t_{k}$ so fine that

$$
\begin{gathered}
f^{\prime \prime}(\bar{u})\left[i_{0}^{\prime}\left(\bar{u}\left(t_{1}\right), \ldots, \bar{u}\left(t_{k}\right)\right)\left(\bar{u}\left(t_{1}\right), \ldots, \bar{u}\left(t_{k}\right)\right),\right. \\
\left.i_{0}^{\prime}\left(\bar{u}\left(t_{1}\right), \ldots, \bar{u}\left(t_{k}\right)\right)\left(\bar{u}\left(t_{1}\right), \ldots, \bar{u}\left(t_{k}\right)\right)\right]<0 .
\end{gathered}
$$

By considering, instead of $\bar{u}, \bar{u}(\cdot+l)$ for some $l \in \mathbf{Z}$, we can suppose that

$$
\left[\frac{-t_{1}}{2}\right]=\left[\frac{t_{k}}{2}\right]=\omega \in \mathbf{N}
$$

Having thus chosen $t_{1}<\ldots<t_{k}$, we can find $\delta \in\left(0, \frac{a}{2}\right)$ such that for any $i \in(1, \ldots, k-1), \alpha \in[0, \delta]$ and for any $(x, y) \in$ $B_{p}\left(\bar{u}\left(t_{i}\right), \delta\right) \times B_{p}\left(\bar{u}\left(t_{i+1}\right), \delta\right)$, problem $P(x, y, \alpha, i)$ has a solution. For $\alpha \in[0, \delta]$ we define the operator

$$
G^{\alpha}: \quad \prod_{i=1}^{k} B_{p}\left(\bar{u}\left(t_{i}\right), \delta\right) \subset \mathbf{R}^{k p} \rightarrow \mathbf{R}^{k p}
$$

by

$$
\begin{aligned}
G^{\alpha}\left(x_{1}, \ldots, x_{k}\right)= & \left(v^{\mathrm{dep}}\left(x_{1}, x_{2}, \alpha, 1\right)\right. \\
& -v_{-\infty}^{\mathrm{arr}}\left(x_{1}, \alpha\right),\left(v^{\mathrm{dep}}\left(x_{2}, x_{3}, \alpha, 2\right)\right. \\
& -v^{\mathrm{arr}}\left(x_{1}, x_{2}, \alpha, 2\right), \ldots, v_{\infty}^{\mathrm{dep}}\left(x_{k}, \alpha\right) \\
& \left.-v^{\mathrm{arr}}\left(x_{k-1}, x_{k}, \alpha, k\right)\right) .
\end{aligned}
$$


By our choice of the partition and of $\delta$ we have that, for $\alpha \in[0, \delta]$,

$$
G^{\alpha}\left(x_{1}, \ldots, x_{k}\right)=0 \Leftrightarrow i_{\alpha}\left(x_{1}, \ldots, x_{k}\right) \text { solves }\left(P^{\boldsymbol{\alpha}}\right) \text {. }
$$

By the continuity of $i_{0}$ and hypothesis $(\star)$, we can suppose that, possibly taking a smaller $\delta$, the only solution of $\left(P^{0}\right)$ contained in the closure of $i_{0}\left(\prod_{i=1}^{k} B_{p}\left(\bar{u}\left(t_{i}\right), \delta\right)\right)$ is $\bar{u}$. This and (1.9) yield that $\inf \left\{\left\|G^{0}\left(x_{1}, \ldots, x_{k}\right)\right\|_{\mathbf{R}^{k p}}:\left(x_{1}, \ldots, x_{k}\right) \in \partial \prod_{i=1}^{k} B_{p}\left(\bar{u}\left(t_{i}\right), \delta\right)\right\}=\chi>0$.

It is well-known that $G^{0}$ is the gradient of

$$
\begin{gathered}
\tilde{f}: \prod_{i=1}^{k} B_{p}\left(\bar{u}\left(t_{i}\right), \delta\right) \rightarrow \mathbf{R} \\
\tilde{f}: \quad\left(x_{1}, \ldots, x_{k}\right) \rightarrow f\left(i_{0}\left(x_{1}, \ldots, x_{k}\right)\right)
\end{gathered}
$$

where $f$ is defined by (1.1). As it is shown in [3], in our hypotheses on $W f$ is of class $C^{3}$; since $i_{0}$ is clearly of class $C^{3}$, it follows that $\tilde{f}$ too is of class $C^{3}$.

Step 2. - We assert that, since $\bar{u}$ is an isolated Mountain Pass point of $f$ and $W 4$ ) holds, it happens

$$
\operatorname{deg}\left(G^{0}, \prod_{i=1}^{k} B_{p}\left(\bar{u}\left(t_{i}\right), \delta\right), 0\right)=-1
$$

Since the argument to prove (1.11) is similar to that of [3], proposition 2.3, we just sketch it. A little calculation shows that, if we define

$$
g\left(x_{1}, \ldots, x_{k}\right)=\tilde{f}^{\prime}\left(x_{1}, \ldots, x_{k}\right)\left(x_{1}, \ldots, x_{k}\right)
$$

then (1.7) implies that

$$
g^{\prime}\left(\bar{u}\left(t_{1}\right), \ldots, \bar{u}\left(t_{k}\right)\right)\left(\bar{u}\left(t_{1}\right), \ldots, \bar{u}\left(t_{k}\right)\right)<0
$$

and thus that

$$
M_{k}=\left\{\left(x_{1}, \ldots, x_{k}\right) \in \prod_{i=1}^{k} B_{p}\left(\bar{u}\left(t_{i}\right), \delta\right): g\left(x_{1}, \ldots, x_{k}\right)=0\right\}
$$

is a codimension 1 manifold in a neighbourhood of $\left(\bar{u}\left(t_{1}\right), \ldots, \bar{u}\left(t_{k}\right)\right)$. 
We assert that $\left(\bar{u}\left(t_{1}\right), \ldots, \bar{u}\left(t_{k}\right)\right)$ is a strict local minimum for $\tilde{f}$ constrained on $M_{k}$. Since $\left(\bar{u}\left(t_{1}\right), \ldots, \bar{u}\left(t_{k}\right)\right)$ is isolated and the critical points of $\left.\tilde{f}\right|_{M_{k}}$ are critical points of $\tilde{f}$, the assertion follows if we show that $\left(\bar{u}\left(t_{1}\right), \ldots, \bar{u}\left(t_{k}\right)\right)$ is a local minimum. But, if there were $\left\{\mathbf{x}_{n}\right\} \subset M_{k}$ with $\mathbf{x}_{n} \rightarrow\left(\bar{u}\left(t_{1}\right), \ldots, \bar{u}\left(t_{k}\right)\right)$ and $\tilde{f}\left(\mathbf{x}_{n}\right)<\tilde{f}\left(\bar{u}\left(t_{1}\right), \ldots, \bar{u}\left(t_{k}\right)\right)$, then by (1.7) and the Taylor formula we would have, for a positive $\varepsilon$ not depending on $n$,

$$
\forall \gamma \in[-\varepsilon, \varepsilon], \quad \tilde{f}\left((1+\gamma) \mathbf{x}_{n}\right)<\tilde{f}\left(\bar{u}\left(t_{1}\right), \ldots, \bar{u}\left(t_{k}\right)\right) .
$$

But in [3] it has been shown that $f(\bar{u})$ is the lowest positive critical level of $f$ and that $f(\lambda \bar{u})$ is increasing for $\lambda \in(0,1)$ and decreasing for $\lambda>1$. It is now easy to construct, using (1.12) and the above considerations, a Mountain pass path for $f$ below $f(\bar{u})$, contradicting the fact that $f(\bar{u})$ is the lowest positive critical level of $f$.

Thus $\left(\bar{u}\left(t_{1}\right), \ldots, \bar{u}\left(t_{k}\right)\right)$ is a strict local minimum for $\left.\tilde{f}\right|_{M_{k}}$ and, as a consequence, we have that, for $\gamma>0$ and small enough, the connected component of

$$
\left\{\mathbf{x} \in M_{k}: \tilde{f}(\mathbf{x})<f(\bar{u})+\gamma\right\}
$$

containing $\left(\bar{u}\left(t_{1}\right), \ldots, \bar{u}\left(t_{k}\right)\right)$ is a $(k p-1)$-cell. Let us call it $A_{\gamma}$. We note that, by (1.7) and the continuity of $\tilde{f}^{\prime \prime}$, for $\gamma$ positive and small enough we have

$$
\tilde{f}^{\prime \prime}(\mathbf{x})(\mathbf{x}, \mathbf{x})<0, \quad \forall \mathbf{x} \in A_{\gamma} .
$$

We now approximate $\tilde{f}$ in the $C^{3}$ topology with a Morse functional $\bar{f}$, and set

$$
\bar{M}_{k}=\left\{\left\langle\bar{f}^{\prime}(\mathbf{x}), \mathbf{x}\right\rangle=0\right\} .
$$

Since $\left(\bar{u}\left(t_{1}\right), \ldots, \bar{u}\left(t_{k}\right)\right)$ is an isolated critical point of $\tilde{f}$, for $\bar{f}$ sufficiently close to $\tilde{f}$, the critical points of $\bar{f}$, which we denote by $\mathbf{y}_{1}, \ldots, \mathbf{y}_{q}$, are close to $\left(\bar{u}\left(t_{1}\right), \ldots, \bar{u}\left(t_{k}\right)\right)$. Moreover, $\bar{A}_{\gamma}$, the connected component of

$$
\left\{\mathbf{x} \in \bar{M}_{k}: \bar{f}(\mathbf{x})<\gamma\right\}
$$

containing them, is a $(k p-1)$-cell. From (1.13), the closeness of $\bar{f}$ with $\tilde{f}$ and of $\mathbf{y}_{1}, \ldots, \mathbf{y}_{q}$ with $\left(\bar{u}\left(t_{1}\right), \ldots, \bar{u}\left(t_{k}\right)\right)$, it follows that

$$
f^{\prime \prime}\left(\mathbf{y}_{i}\right)\left(\mathbf{y}_{i}, \mathbf{y}_{i}\right)<0, \quad \forall i \in(1, \ldots, q) .
$$

It is moreover easy to see that from the above formula and the fact that $\bar{f}^{\prime \prime}\left(\mathbf{y}_{i}\right) \mathbf{y}_{i}$ is normal to $\bar{M}_{k}$ in $\mathbf{y}_{i}$, that $\mathbf{y}_{i}$ is nondegenerate also for $\left.\bar{f}\right|_{\bar{M}_{k}}$. 
We denote by $m_{i}$ the Morse index of $\left.\bar{f}\right|_{\bar{M}_{k}}$ in $\mathbf{y}_{i}$. Since $\bar{A}_{\gamma}$ is a $(k p-1)$-cell, we get from the Morse-Bott formula that

$$
\sum_{i=1}^{q}(-1)^{m_{i}}=1
$$

By (1.13), $\mathbf{y}_{i}$ has Morse index $m_{i}+1$ in $\mathbf{R}^{k p}$, and thus

$$
\operatorname{deg}\left(\bar{f}, \prod_{i=1}^{k} B_{p}\left(\bar{u}\left(t_{i}\right), \delta\right), 0\right)=\sum_{i=1}^{q}(-1)^{m_{i}+1}=-1
$$

Since $\bar{f}$ and $\tilde{f}$ are $C^{3}$ close, the last formula implies (1.11).

Step 3. - We are now going to glue together the solutions of $\left(P^{0}\right)$. We suppose at first that the sequence $\left\{m_{i}\right\}_{i=0}^{l}$ is finite. In the following, $\delta$ will be supposed so small that $0 \notin B_{p}\left(\bar{u}\left(t_{i}\right), \delta\right)$ for $i=1, k$.

Let us consider $\Phi_{\alpha}$ in $B_{p}(0, a) \times B_{p}(0, a)$; we define a foliation $F^{s}$ whose leaves are $F_{x}^{s}=\left\{(x, y), y \in \mathbf{R}^{p}\right\}$ for $x \in B_{p}\left(\bar{u}\left(t_{k}\right), \delta\right)$ and a foliation $F^{u}$ whose leaves are $F_{\tilde{x}}^{u}=\left\{(\tilde{x}, y), y \in \mathbf{R}^{p}\right\}$ for $\tilde{x} \in B_{p}\left(\bar{u}\left(t_{1}\right), \delta\right)$. By our choice of $a, F^{s}$ is transversal to the stable, and $F^{u}$ to the unstable manifold of $\Phi_{\alpha}$ at 0 . It is a consequence of the $\lambda$ lemma (see for instance [12]) that there is $\bar{N} \in \mathbf{N}$ such that, for $n_{1}, n_{2} \geq \bar{N}$ and $\alpha \in\left[0, \alpha_{0}\right]$, each leaf $\Phi_{\alpha}^{n_{1}}\left(F_{x}^{s}\right)$ intersects any other leaf $\Phi_{\alpha}^{-n_{2}}\left(F_{\tilde{x}}^{u}\right)$ in a point $q(x, \tilde{x}, \alpha)$ depending continuously on $(x, \tilde{x}, \alpha)$. This implies that, for $m \geq 2 \bar{N}, \alpha \in\left[0, \alpha_{0}\right],(x, \tilde{x}) \in B_{p}\left(\bar{u}\left(t_{k}\right), \delta\right) \times B_{p}\left(\bar{u}\left(t_{1}\right), \delta\right)$, there are $v_{m}^{\text {dep }}(x, \tilde{x}, \alpha)$ and $v_{m}^{\text {arr }}(x, \tilde{x}, \alpha)$, depending continuously on their variables, such that the solution of

$$
\left\{\begin{array}{c}
\ddot{u}=-V^{\prime}(u, t)-\alpha \dot{u} \\
u\left(t_{k}\right)=x \\
\dot{u}\left(t_{k}\right)=v_{m}^{\text {dep }}(x, \tilde{x}, \alpha)
\end{array}\right.
$$

satisfies $u\left(t_{k}+m\right)=\tilde{x}, \dot{u}\left(t_{k}+m\right)=v_{m}^{\text {arr }}(x, \tilde{x}, \alpha)$. Also by the $\lambda$-lemma it follows in a standard way that

$$
\begin{gathered}
\exists N_{0} \geq 2 \bar{N}: \quad \forall(x, \tilde{x}) \in B_{p}\left(\bar{u}\left(t_{k}\right), \delta\right) \times B_{p}\left(\bar{u}\left(t_{1}\right), \delta\right), \\
\forall \alpha \in\left[0, \alpha_{0}\right], \quad \forall m \geq N_{0} \\
\left|v_{m}^{\text {dep }}(x, \tilde{x}, \alpha)-v_{\infty}^{\text {dep }}(x, \alpha)\right| \\
+\left|v_{m}^{\text {arr }}(x, \tilde{x}, \alpha)-v_{-\infty}^{\text {arr }}(\tilde{x}, \alpha)\right|<\frac{\chi}{8}
\end{gathered}
$$


where $\chi$ is defined by (1.10). We now set

$$
\mathbf{x}^{i}=\left(x_{1}^{i}, \ldots, x_{k}^{i}\right) \in \mathbf{R}^{k p}
$$

Given the strictly increasing sequence $\left\{m_{i}\right\}_{i=0}^{l} \subset \mathbf{N}$, with $m_{i}-m_{i-1}-$ $\left(t_{k}-t_{1}\right) \geq N_{0}$, we set $n_{i}=m_{i}-m_{i-1}-\left(t_{k}-t_{1}\right)$ for $i \in(1, \ldots, l)$ and we consider the operator

$$
G_{l}^{\alpha}: \prod_{j=0}^{l} \prod_{i=1}^{k} B_{p}\left(\bar{u}\left(t_{i}\right), \delta\right) \subset \mathbf{R}^{(l+1) k p} \rightarrow \mathbf{R}^{(l+1) k p}
$$

defined by

$$
\begin{aligned}
G_{l}^{\alpha}\left(\mathbf{x}^{0}, \ldots, \mathbf{x}^{l}\right)= & v^{\mathrm{dep}}\left(x_{1}^{0}, x_{2}^{0}, \alpha, 1\right) \\
& -v_{-\infty}^{\operatorname{arr}}\left(x_{1}^{0}, \alpha\right) v^{\mathrm{dep}}\left(x_{2}^{0}, x_{3}^{0}, \alpha, 2\right) \\
& -v^{\operatorname{arr}}\left(x_{1}^{0}, x_{2}^{0}, \alpha, 2\right), \ldots, v_{n_{1}}^{\text {dep }}\left(x_{k}^{0}, x_{1}^{1}, \alpha\right) \\
& -v^{\operatorname{arr}}\left(x_{k-1}^{0}, x_{k}^{0}, \alpha, k\right), v^{\text {dep }}\left(x_{1}^{1}, x_{2}^{1}, \alpha, 1\right) \\
& -v_{n_{1}}^{\operatorname{arr}}\left(x_{k}^{0}, x_{1}^{1}, \alpha\right), v^{\text {dep }}\left(x_{2}^{1}, x_{3}^{1}, \alpha, 2\right) \\
& -v^{\operatorname{arr}}\left(x_{1}^{1}, x_{2}^{1}, \alpha, 2\right), \ldots, v_{\infty}^{\text {dep }}\left(x_{k}^{l}, \alpha\right) \\
& \left.-v^{\operatorname{arr}}\left(x_{k-1}^{l}, x_{k}^{l}, \alpha, k\right)\right)
\end{aligned}
$$

which we can also write as

$$
\begin{aligned}
G_{l}^{\alpha}\left(\mathbf{x}^{0}, \ldots, \mathbf{x}^{l}\right)= & \left(G^{\alpha}\left(\mathbf{x}^{0}\right), G^{\alpha}\left(\mathbf{x}^{1}\right), \ldots, G^{\alpha}\left(\mathbf{x}^{l}\right)\right) \\
& +\left(0, \ldots, 0, v_{n_{1}}^{\mathrm{dep}}\left(x_{k}^{0}, x_{1}^{1}, \alpha\right)\right. \\
& -v_{\infty}^{\mathrm{dep}}\left(x_{k}^{0}, \alpha\right), v_{-\infty}^{\text {arr }}\left(x_{1}^{1}, \alpha\right) \\
& -v_{n_{1}}^{\text {arr }}\left(x_{k}^{0}, x_{1}^{1}, \alpha\right), 0, \ldots, v_{n_{l}}^{\text {dep }}\left(x_{k}^{l-1}, x_{1}^{l}, \alpha\right) \\
& -v_{\infty}^{\mathrm{dep}}\left(x_{k}^{l-1}, \alpha\right), v_{-\infty}^{\operatorname{arr}}\left(x_{1}^{l}, \alpha\right) \\
& \left.-v_{n_{l}}^{\text {arr }}\left(x_{k}^{l-1}, x_{1}^{l}, \alpha\right), 0, \ldots, 0\right) .
\end{aligned}
$$

We now define

$$
i_{l}^{\alpha}: \prod_{j=0}^{l} \prod_{i=1}^{k} B_{p}\left(\bar{u}\left(t_{i}\right), \delta\right) \rightarrow H^{1}\left(\mathbf{R}, \mathbf{R}^{p}\right)
$$


by

$$
\begin{aligned}
i_{l}^{\alpha}: & \left(\mathbf{x}^{0}, \ldots, \mathbf{x}^{l}\right) \rightarrow u(t) \\
= & \left\{\begin{aligned}
& u_{0}^{0}(t), \quad t \leq m_{0}+t_{1} \\
& u_{i}^{j}(t), m_{j}+t_{i} \leq \begin{array}{l}
t \leq m_{j}+t_{i+1}, \quad(i, j) \neq(k, l),(0,0) \\
u_{k}^{l}(t), \quad t \geq m_{l}+t_{k}
\end{array}
\end{aligned}\right.
\end{aligned}
$$

where $u_{0}^{0}\left(\cdot+m_{0}\right)$ solves $P\left(t_{1}, x_{1}^{0}, \alpha,-\infty\right), u_{i}^{j}\left(\cdot+m_{j}\right)$ solves $P\left(x_{i}^{j}, x_{i+1}^{j}, \alpha, i\right)$ for $1 \leq i \leq k-1$ and $0 \leq j \leq l, u_{k}^{j}\left(\cdot+m_{j}\right)$ solves $\tilde{P}_{n_{j+1}}\left(x_{k}^{j}, x_{1}^{j+1}, \alpha\right)$ for $0 \leq j<l$ and $u_{k}^{l}\left(\cdot+m_{l}\right)$ solves $P\left(t_{k}, x_{k}^{l}, \alpha, \infty\right)$. It is easy to see that $i_{l}^{\alpha}$ is continuous and that

$$
\left.\begin{array}{c}
\alpha \in[0, \delta] \\
G_{l}^{\alpha}\left(\mathbf{x}^{0}, \ldots, \mathbf{x}^{l}\right)=0 \Leftrightarrow i_{l}^{\alpha}\left(\mathbf{x}^{0}, \ldots, \mathbf{x}^{l}\right) \text { solves }\left(P^{\alpha}\right)
\end{array}\right\}
$$

We now note that, if

$$
\left(\mathbf{x}^{0}, \ldots, \mathbf{x}^{l}\right) \in \partial \prod_{j=0}^{l} \prod_{i=1}^{k} B_{p}\left(\bar{u}\left(t_{i}\right), \delta\right)
$$

then $\mathbf{x}^{j} \in \partial \prod_{i=1}^{k} B_{p}\left(\bar{u}\left(t_{i}\right), \delta\right)$ for some $j \in(0, \ldots, l)$. From (1.10) and (1.14) it follows

$$
\begin{aligned}
& \inf \left\{\| G^{0}\left(\mathbf{x}^{j}\right)-\lambda\left(v_{-\infty}^{\operatorname{arr}}\left(x_{1}^{j}, 0\right)\right.\right. \\
& \left.-v_{n_{j}}^{\operatorname{arr}}\left(x_{k}^{j-1}, x_{1}^{j}, 0\right), 0, \ldots, 0, v_{n_{j+1}}^{\text {dep }}\left(x_{k}^{j}, x_{1}^{j+1}, 0\right)-v_{\infty}^{\text {dep }}\left(x_{k}^{j}, 0\right)\right) \|_{\mathbf{R}^{k p}}: \\
& \left.\mathbf{x}^{j} \in \partial \prod_{i=1}^{k} B_{p}\left(\bar{u}\left(t_{i}\right), \delta\right), \lambda \in[0,1]\right\} \geq \frac{\chi}{2} .
\end{aligned}
$$

The previous considerations and the orthogonality of the various $\mathbf{R}^{k p}$ in $\mathbf{R}^{(l+1) k p}$ imply

$$
\begin{aligned}
\inf & \left\{\| G_{l}^{0}\left(\mathbf{x}^{0}, \ldots, \mathbf{x}^{l}\right)\right. \\
& -\lambda\left(0, \ldots, 0, v_{n_{1}}^{\text {dep }}\left(x_{k}^{0}, x_{1}^{1}, 0\right)-v_{\infty}^{\text {dep }}\left(x_{k}^{0}, 0\right), v_{-\infty}^{\text {arr }}\left(x_{1}^{1}, 0\right)\right. \\
& -v_{n_{1}}^{\text {arr }}\left(x_{k}^{0}, x_{1}^{1}, 0\right), 0, \ldots, v_{n_{l}}^{\text {dep }}\left(x_{k}^{l-1}, x_{1}^{l}, 0\right) \\
& -v_{\infty}^{\text {dep }}\left(x_{k}^{l-1}, 0\right), v_{-\infty}^{\text {arr }}\left(x_{1}^{l}, 0\right)
\end{aligned}
$$

Vol. $12, n^{\circ} 1-1995$. 


$$
\begin{aligned}
& \left.-v_{n_{l}}^{\mathrm{arr}}\left(x_{k}^{l-1}, x_{1}^{l}, 0\right), 0, \ldots, 0\right) \|_{\mathbf{R}^{(l+1) k p}}: \\
& \left.\left(\mathbf{x}^{0}, \ldots, \mathbf{x}^{l}\right) \in \partial \prod_{j=0}^{l} \prod_{i=1}^{k} B_{p}\left(\bar{u}\left(t_{i}\right), \delta\right), \lambda \in[0,1], l \in \mathbf{N}\right\} \geq \frac{\chi}{2} .
\end{aligned}
$$

By (1.15), (1.17) and the homotopy property of the degree we have that

$$
\begin{aligned}
& \operatorname{deg}\left(G_{l}^{0}, \prod_{j=0}^{l} \prod_{i=1}^{k} B_{p}\left(\bar{u}\left(t_{i}\right), \delta\right), 0\right) \\
& =\operatorname{deg}\left(\left(G^{0}, G^{0}, \ldots, G^{0}\right), \prod_{j=0}^{l} \prod_{i=1}^{k} B_{p}\left(\vec{u}\left(t_{i}\right), \delta\right), 0\right)
\end{aligned}
$$

which by the formula for the degree of a product of maps and (1.11) implies

$$
\operatorname{deg}\left(G_{l}^{0}, \prod_{j=0}^{l} \prod_{i=1}^{k} B_{p}\left(\bar{u}\left(t_{i}\right), \delta\right), 0\right)=(-1)^{l+1} .
$$

Moreover, (1.10) and the continuity of $G^{\alpha}$ in the $\alpha$ variable imply the existence of $\alpha(\delta) \in(0, \delta]$, only depending on $\delta$, such that

$$
\inf _{\alpha \in[0, \alpha(\delta)]} \inf \left\{\left\|G^{\alpha}(\mathbf{x})\right\|_{\mathbf{R}^{k p}}: \mathbf{x} \in \partial \prod_{i=1}^{k} B_{p}\left(\bar{u}\left(t_{i}\right), \delta\right)\right\} \geq \frac{\chi}{2}
$$

The same argument which we have used to get (1.17) now yields

$$
\begin{aligned}
& \inf _{\substack{l \in \mathbf{N} \\
\inf _{\alpha \in[0, \alpha(\delta)]}}} \inf \left\{\left\|G_{l}^{\alpha}\left(\mathbf{x}^{0}, \ldots, \mathbf{x}^{l}\right)\right\|_{\mathbf{R}^{(l+1) k_{p}}}:\right. \\
& \left.\left(\mathbf{x}^{0}, \ldots, \mathbf{x}^{l}\right) \in \partial \prod_{j=0}^{l} \prod_{i=1}^{k} B_{p}\left(\bar{u}\left(t_{i}\right), \delta\right)\right\} \geq \frac{\chi}{4}
\end{aligned}
$$

Formulae (1.18) and (1.19) now imply (see for instance [8]) the existence of a continuum $\theta \subset \mathbf{R}^{(l+1) k p} \times[0, \alpha(\delta)]$ contained in $\prod_{j=0}^{l} \prod_{i=1}^{k} B_{p}\left(\bar{u}\left(t_{i}\right), \delta\right)$ and connecting $\mathbf{R}^{(l+1) k p} \times\{0\}$ with $\mathbf{R}^{(l+1) k p} \times\{\alpha(\delta)\}$. We note that $\alpha(\delta)$ does not depend on $l$.

We assert that $\Theta_{\left\{m_{i}\right\}}=i_{l}^{\alpha}(\theta)$ is the continuum we are looking for. 
Clearly, it connects $H^{1}\left(\mathbf{R}, \mathbf{R}^{p}\right) \times\{0\}$ with $H^{1}\left(\mathbf{R}, \mathbf{R}^{p}\right) \times\{\alpha(\delta)\}$ and its elements are solutions of $\left(P^{\alpha}\right)$ by (1.16). it remains to show (1.2).

Step 4. - To show (1.2) it is sufficient to show that $\forall \varepsilon>0$ there is $\bar{\delta} \in(0, \delta), N_{1} \geq N_{0}$ and $\bar{\alpha} \in(0, \alpha(\bar{\delta})]$ such that

$$
\left.\begin{array}{c}
\forall N \geq N_{1}, \\
\forall l \in \mathbf{N}, \quad \forall\left\{m_{j}\right\}_{j=0}^{l}, m_{j}-m_{j-1}-\left(t_{k}-t_{1}\right) \geq 2 N, \\
\forall\left(\mathbf{x}^{0}, \ldots, \mathbf{x}^{l}\right) \in \prod_{j=0}^{l} \prod_{i=1}^{k} B_{p}\left(\bar{u}\left(t_{i}\right), \bar{\delta}\right), \\
\forall \alpha \in[0, \bar{\alpha}], \quad \forall j \in(0, \ldots, l), \\
\left\|i_{\alpha}^{l}\left(\mathbf{x}^{0}, \ldots, \mathbf{x}^{l}\right)\left(\cdot+m_{j}\right) \phi_{\omega+N}-\bar{u}\right\|_{H^{1}\left(\mathbf{R}, \mathbf{R}^{p}\right)}^{2} \leq \varepsilon
\end{array}\right\}
$$

where $\omega$ is the same as in formula (1.8). it is clear that (1.20) implies that the zero of $G_{l}^{\alpha}$ belonging to $\prod_{j=0}^{l} \prod_{i=1}^{k} B_{p}\left(\bar{u}\left(t_{i}\right), \bar{\delta}\right)$, and whose existence follows by the previous arguments, satisfies (1.2). We note that by definition of $i_{\alpha}^{l}$ we have

$$
\begin{aligned}
& \left(\mathbf{x}^{0}, \ldots, \mathbf{x}^{l}\right) \in \prod_{j=0}^{l} \prod_{i=1}^{k} B_{p}\left(\bar{u}\left(t_{i}\right), \bar{\delta}\right) \\
& \quad \Rightarrow\left|i_{\alpha}^{l}\left(\mathbf{x}^{0}, \ldots, \mathbf{x}^{l}\right)\left(t_{i}+m_{j}\right)-\bar{u}\left(t_{i}\right)\right|_{\mathbf{R}^{k p}}<\bar{\delta}, \quad \forall i, j
\end{aligned}
$$

and that $i_{\alpha}^{l}\left(\mathbf{x}^{0}, \ldots, \mathbf{x}^{l}\right)(\cdot)=w$ solves $\left(R^{\alpha}\right)$ in any interval $\left(t_{i}, t_{i+1}\right)$. Thus it follows easily that, for $\bar{\delta}$ and $\bar{\alpha}$ positive and small enough, we have

$$
\left.\begin{array}{c}
\forall \alpha \in[0, \bar{\alpha}], \quad \forall j, \\
\int_{\left[t_{1}, t_{k}\right]}\left[\left|\dot{w}\left(\cdot+m_{j}\right)-\dot{\bar{u}}\right|^{2}+\left|w\left(\cdot+m_{j}\right)-\bar{u}\right|^{2}\right] d t \leq \frac{\varepsilon}{2} .
\end{array}\right\}
$$

We now note that by (1.14) we can take $N_{1}$ so big that, for $N \geq N_{1},\left|\left(w\left(t_{k}+m_{j}\right), \dot{w}\left(t_{k}+m_{j}\right)\right)-\left(\bar{u}\left(t_{k}\right), \dot{\bar{u}}\left(t_{k}\right)\right)\right| \leq \bar{\delta}$, that $w\left(\left[t_{k}+m_{j}, t_{k}+m_{j}+N\right]\right) \subset B(0, a)$ and that $w\left(t_{k}+m_{j}+N\right) \subset B_{p}(0, \bar{\delta})$. We assert that there is $N_{1} \in \mathbf{N}, \bar{\delta}>0$ and $\bar{\alpha}>0$ such that for any $\alpha \in[0, \bar{\alpha}]$, and $N \geq N_{1}$, for any $z$ solving $R^{\alpha}$ and satisfying $\left|\left(z\left(t_{k}\right), \dot{z}\left(t_{k}\right)\right)-\left(\bar{u}\left(t_{k}\right), \dot{\bar{u}}\left(t_{k}\right)\right)\right| \leq \bar{\delta}, z\left(\left[t_{k}, t_{k}+N\right]\right) \subset B(0, a)$ and $z\left(t_{k}+N\right) \in B_{p}(0, \bar{\delta})$, it happens

$$
\int_{t_{k}}^{t_{k}+N}|\dot{z}-\dot{\bar{u}}|^{2}+|z-\bar{u}|^{2} d t \leq \frac{\varepsilon}{4} .
$$


Indeed, if (1.22) is not true, then we find $N_{s} \rightarrow \infty, \bar{\delta}_{s} \rightarrow 0, \bar{\alpha}_{s} \rightarrow 0$ and $z_{s}$ satisfying the above conditions for $N_{s}$ and $\bar{\delta}_{s}$ and $\bar{\alpha}_{s}$ such that

$$
\int_{t_{k}}^{t_{k}+N_{s}}\left|\dot{z}_{s}-\dot{\bar{u}}\right|^{2}+\left|z_{s}-\bar{u}\right|^{2} d t>\frac{\varepsilon}{4} .
$$

But now a concentration-compactness argument similar to that in [3] shows that $\left\{\left.z_{s}\right|_{\left[t_{k}, t_{k}+N_{s}\right]}\right\}$ converges in the $H^{1}$ topology to $\left.\bar{u}\right|_{\left[t_{k}, \infty\right)}$, a contradiction with the last formula. Thus (1.22) implies that, for $\bar{\delta}$ and $\bar{\alpha}$ small enough and for $N$ big enough, we have

$$
\left.\begin{array}{c}
\forall j, \quad \forall \alpha \in[0, \bar{\alpha}], \\
\int_{\left[t_{k}, t_{k}+N\right]}\left[\left|\dot{w}\left(\cdot+m_{j}\right)-\dot{\bar{u}}\right|^{2}+\left|w\left(\cdot+m_{j}\right)-\bar{u}\right|^{2}\right] d t \leq \frac{\varepsilon}{4} .
\end{array}\right\}
$$

Analogously one shows that for $\bar{\delta}$ small enough and for $N$ big enough,

$$
\left.\begin{array}{c}
\forall j, \quad \forall \alpha \in[0, \bar{\alpha}], \\
\int_{\left[t_{1}-N, t_{1}\right]}\left[\left|\dot{w}\left(\cdot+m_{j}\right)-\dot{\bar{u}}\right|^{2}+\left|w\left(\cdot+m_{j}\right)-\bar{u}\right|^{2}\right] d t \leq \frac{\varepsilon}{4} \cdot
\end{array}\right\}
$$

Formula (1.20) now follows summing up (1.21), (1.23) and (1.24).

If $\left\{m_{j}\right\}_{j \in \mathbf{Z}}$ is infinite, the theorem follows as in [14] or in [2]: we truncate $\left\{m_{j}\right\}_{j \in \mathbf{Z}}$ to $\left\{m_{j}\right\}_{j=-l}^{l}$, find the corresponding $\Theta_{\left\{m_{j}\right\}_{j=-l}^{l}}$ and then get $\Theta_{\left\{m_{j}\right\}_{j \in z}}$ as a limit when $l$ goes to infinity. We do not repeat this procedure, since it is already contained in the above references.

If $\left\{m_{j}\right\}_{j \in \mathbf{Z}}$ is periodic, say of period $l$, the branch of periodic solutions can be found applying the previous arguments to the operator

$$
\begin{aligned}
\bar{G}_{l}^{\alpha}\left(\mathbf{x}^{1}, \ldots, \mathbf{x}^{l}\right)= & \left(v^{\mathrm{dep}}\left(x_{1}^{1}, x_{2}^{1}, \alpha, 1\right)\right. \\
& -v_{n_{l}}^{\operatorname{arr}}\left(x_{k}^{l}, x_{1}^{1}, \alpha\right), v^{\text {dep }}\left(x_{2}^{1}, x_{3}^{1}, \alpha, 2\right) \\
& -v^{\operatorname{arr}}\left(x_{1}^{1}, x_{2}^{1}, \alpha, 2\right), \ldots, v_{n_{1}}^{\text {dep }}\left(x_{k}^{1}, x_{1}^{2}, \alpha\right) \\
& -v^{\operatorname{arr}}\left(x_{k-1}^{1}, x_{k}^{1}, \alpha\right), v^{\text {dep }}\left(x_{1}^{2}, x_{2}^{2}, \alpha, 1\right) \\
& -v_{n_{1}}^{\operatorname{arr}}\left(x_{k}^{1}, x_{1}^{2}, \alpha\right), v^{\text {dep }}\left(x_{2}^{2}, x_{3}^{2}, \alpha, 2\right) \\
& -v^{\operatorname{arr}}\left(x_{1}^{2}, x_{2}^{2}, \alpha, 2\right), \ldots, v_{n_{l}}^{\text {dep }}\left(x_{k}^{l}, x_{1}^{1}, \alpha\right) \\
& \left.-v^{\operatorname{arr}}\left(x_{k-1}^{l}, x_{k}^{l}, \alpha, k\right)\right)
\end{aligned}
$$

whose zeroes correspond to solutions of $\left(R^{\alpha}\right)$ of period $m_{l}-m_{0}$. The arguments just used give us the result also in this case. 
With the same arguments as in [14], the above theorem implies the following corollary.

COROLLARY 1.2. - Let W1-4) and ( $\star$ ) hold. Then there is $\bar{\alpha}>0$ such that, for $\alpha \in[0, \bar{\alpha}], \Phi_{\alpha}$ has positive topological entropy.

\section{SECTION 2}

We begin this section recalling what is usually meant by "infinite cascade of period-doubling bifurcations" for $\left(R^{\alpha}\right)$. A branch of periodic solutions of $\left(R^{\alpha}\right)$ of, say, period $m$, embeds naturally into a branch of periodic solutions of $\left(R^{\alpha}\right)$ of period $2 \mathrm{~m}$. If this latter branch contains solutions of minimal period $2 m$, we say that a period-doubling bifurcation has occurred. If the branch of period $2 m$ is again subset a branch of period $4 m$ which contains solutions of minimal period $4 \mathrm{~m}$, and so on ad infinitum, we speak of an infinite cascade of period-doubling bifurcations.

We recall that, by lemma 1.4 of [3], there is $\alpha_{0}>0$ such that $\left(P^{\alpha}\right)$ has no solutions for $\alpha>\alpha_{0}$. We have seen in section 1 that a Mountainpass solution $\bar{u}$ of $\left(P^{0}\right)$ is contained, if $(\star)$ holds, in a connected closed set $\Theta_{\{0\}} \subset H^{1}\left(\mathbf{R}, \mathbf{R}^{p}\right) \times[0, \bar{\alpha}]$ which is not reduced to the only $\bar{u}$. We denote by $\Gamma \subset H^{1}\left(\mathbf{R}, \mathbf{R}^{p}\right) \times\left[0, \alpha_{0}\right)$ the maximal continuum containing $\Theta_{\{0\}}$. For $u \in H^{1}\left(\mathbf{R}, \mathbf{R}^{p}\right)$ and $a$ the same as in section 1 , we set

$$
\begin{aligned}
& \underline{t}(u)=\sup \left\{n \in \mathbf{Z}: u((-\infty, n]) \subset B_{p}\left(0, \frac{a}{2}\right)\right\} \\
& \bar{t}(u)=\inf \left\{n \in \mathbf{Z}: u([n, \infty)) \subset B_{p}\left(0, \frac{a}{2}\right)\right\} .
\end{aligned}
$$

By the arguments at the beginning of section 1, we have that any solution $u$ of $\left(P^{\alpha}\right)$ satisfies $\|u\|_{\infty} \geq a$, and that the above quantitites are finite. In the proof of theorem 2.5 of [3] it has been shown that, if $\Gamma$ is bounded and $(\star \star)$ holds, then for any $\alpha \geq 0$ there is $\varepsilon(\alpha)>0$ such that any connected component $\Theta$ of $\Gamma \cap H^{1}\left(\mathbf{R}, \mathbf{R}^{p}\right) \times[\alpha-\varepsilon(\alpha), \alpha+\varepsilon(\alpha)]$ satisfies

$$
\begin{gathered}
\sup \{\max (|\bar{t}(u)-\bar{t}(w)|,|\underline{t}(u)-\underline{t}(w)|): \\
(\alpha, u),(\beta, w) \in \Theta\} \leq 2 .
\end{gathered}
$$

Moreover, from lemma 1.1 of [3] it follows that, if $A \subset H^{1}\left(\mathbf{R}, \mathbf{R}^{p}\right) \times$ $\left[0, \alpha_{0}\right]$ is a bounded closed set of solutions of $\left(P^{\alpha}\right)$, it is compact if and 
only if

$$
\sup \{\max (|\bar{t}(u)-\bar{t}(w)|,|\underline{t}(u)-\underline{t}(w)|):(\alpha, u),(\beta, w) \in A\}<\infty
$$

An essential hypothesis in all these considerations is that $\Gamma$ be bounded. In [3] we gave an example of a potential $V \in C^{3}(\mathbf{R} \times \mathbf{R}, \mathbf{R})$ for which all branches of homoclinics are bounded. We do not know whether this holds for a general $V \in C^{3}(\mathbf{R} \times \mathbf{R}, \mathbf{R})$ satisfying $\left.W 1-5\right)$.

THEOREM 2.1. - Let W1-5), ( $\star$ ) and ( $\star \star)$ hold, and let the branch $\Gamma$ defined above be bounded. Then

i) $\exists\left(\tilde{u}, \alpha_{1}\right) \in \Gamma$ with $\alpha_{1}>0$ and such that $\{(\tilde{u}(n), \dot{\tilde{u}}(n))\}$ are, for $n \in \mathbf{Z}$, points of non-transversal homoclinic intersection for $\Phi_{\alpha_{1}}$.

ii) If there is $\nu_{s} \searrow 0$ such that all intersections of the stable and unstable manifold of $\Phi_{\alpha_{1}-\nu_{s}}$ are transversal, then there is an infinite cascade of period-doubling bifurcations near $\tilde{u}$ as $\alpha$ passes above $\alpha_{1}$.

Proof. - We note that by (2.1) we can find a partition $0=\gamma_{0}<\gamma_{1}<$ $\ldots<\gamma_{q}=\alpha_{0}$ of $\left[0, \alpha_{0}\right]$ such that for any $i \in(0, \ldots, q-1)$, any connected component $\Theta$ of $\Gamma \cap H^{1}\left(\mathbf{R}, \mathbf{R}^{p}\right) \times\left[\gamma_{i}, \gamma_{i+1}\right]$ satisfies

$$
\begin{gathered}
\sup \{\max (|\bar{t}(u)-\bar{t}(w)|,|\underline{t}(u)-\underline{t}(w)|): \\
(\alpha, u),(\beta, w) \in \Theta\} \leq 2 .
\end{gathered}
$$

We now take $\Theta_{0}$ to be the subcontinuum of $\Gamma \cap H^{1}\left(\mathbf{R}, \mathbf{R}^{p}\right) \times\left[0, \gamma_{1}\right]$ containing $(\bar{u}, 0)$ and define $\Theta_{i}$ inductively as the union of the connected components of $\Gamma \cap H^{1}\left(\mathbf{R}, \mathbf{R}^{p}\right) \times\left[\gamma_{i}, \gamma_{i+1}\right]$ which intersect $\Theta_{i-1}$. We set

$$
\tilde{\Gamma}=\bigcup_{i=0}^{q-1} \Theta_{i} .
$$

Clearly, $\tilde{\Gamma}$ is a closed connected subset of $\Gamma$, which by (2.2) satisfies

$$
\begin{gathered}
\sup \{\max (|\bar{t}(u)-\bar{t}(w)|,|\underline{t}(u)-\underline{t}(w)|): \\
(\alpha, u),(\beta, w) \in \tilde{\Gamma}\} \leq 2(q+1)
\end{gathered}
$$

and thus by the considerations at the beginning of this section is compact.

Given a set $A \subset \mathbf{R}^{k p} \times \mathbf{R}$, we denote by $V_{\delta}(A)$ its uniform $\delta$ neighbourhood. Given a partition of $\mathbf{R} t_{1}<\ldots<t_{k}$ we define

$$
j_{\left\{t_{i}\right\}}: \quad H^{1}\left(\mathbf{R}, \mathbf{R}^{p}\right) \times \mathbf{R} \rightarrow \mathbf{R}^{k p} \times \mathbf{R}
$$


by

$$
j_{\left\{t_{i}\right\}}: \quad(u, \alpha) \rightarrow\left(u\left(t_{1}\right), \ldots, u\left(t_{k}\right), \alpha\right) .
$$

Since $\tilde{\Gamma}$ is compact, by the methods of section 1 we can find a partition $t_{1}<\ldots<t_{k}$ with $t_{1}, t_{k} \in \mathbf{Z}$ and $\delta \in\left(0, \frac{a}{2}\right)$ such that for any $\alpha \in\left[0, \alpha_{0}\right]$ the operator

$$
G^{\alpha}: V_{\delta}\left(j_{\left\{t_{i}\right\}}(\tilde{\Gamma})\right) \cap \mathbf{R}^{k p} \times\{\alpha\} \rightarrow \mathbf{R}^{k p}
$$

defined as in section 1 is continuous and has as zeroes solutions of $\left(P^{\alpha}\right)$. Indeed, always by compactness of $\tilde{\Gamma}$, as in section 1 we can require $t_{1}<\ldots<t_{k}$ to be so fine that, for any $(u, \alpha) \in \tilde{\Gamma}$, there are no Jacobi fields between $u\left(t_{i}\right)$ and $u\left(t_{i+1}\right)$ and that

$$
\forall(u, \alpha) \in \tilde{\Gamma}, \quad u\left(\left(-\infty, t_{1}\right]\right) \cup u\left(\left[t_{k}, \infty\right)\right) \subset B_{p}\left(0, \frac{a}{2}\right) .
$$

As in section 1, we also have that the functions $v_{m}^{\text {arr }}\left(x_{k}, y_{1}, \alpha\right)$ and $v_{m}^{\text {dep }}\left(x_{k}, y_{1}, \alpha\right)$ are defined for $\mathbf{x}, \mathbf{y} \in V_{\delta}\left(j_{\left\{t_{i}\right\}}(\tilde{\Gamma})\right)$ and $m$ big enough and that, by the $\lambda$-lemma,

$$
\begin{aligned}
& \sup \left\{\left|v_{m}^{\text {dep }}\left(x_{k}, y_{1}, \alpha\right)-v_{\infty}^{\text {dep }}\left(x_{k}, \alpha\right)\right|\right. \\
& \quad+\left|v_{m}^{\text {arr }}\left(x_{k}, y_{1}, \alpha\right)-v_{-\infty}^{\text {arr }}\left(y_{1}, \alpha\right)\right|: \\
& \left.\quad(\mathbf{x}, \alpha),(\mathbf{y}, \alpha) \in V_{\delta}\left(j_{\left\{t_{i}\right\}}(\tilde{\Gamma})\right)\right\} \rightarrow 0 \text { as } m \rightarrow \infty
\end{aligned}
$$

In the following, to shorten the notation, we will drop the $j_{\left\{t_{i}\right\}}$ when thinking of $\tilde{\Gamma}$ and $\Theta_{i}$ as sets in $\mathbf{R}^{k p} \times \mathbf{R}$. We now define

$$
\alpha_{1}=\sup \{\alpha:(u, \alpha) \in \tilde{\Gamma}\} .
$$

We are going to show that $\alpha_{1}$ satisfies the thesis of the theorem.

By theorem 1.1, we have that $\alpha_{1}>0$. We also have that $\alpha_{1}<\alpha_{0}$ : otherwise, $\left(P^{\alpha}\right)$ would have a solution for $\alpha \geq \alpha_{0}$, which we known to be impossible. Since $\tilde{\Gamma}$ is compact, $\alpha_{1}$ is actually a maximum and we can find $\left(\tilde{u}, \alpha_{1}\right) \in \tilde{\Gamma}$.

We assert that, for $n \in \mathbf{Z},(\tilde{u}(n), \dot{\tilde{u}}(n))$ is a point of homoclinic tangency for the stable and unstable manifolds of $\Phi_{\alpha_{1}}$. It is sufficient to show that, if the two manifolds are transversal in $(\tilde{u}(n), \dot{\tilde{u}}(n))$, then $D G^{\alpha_{1}}\left(\tilde{u}\left(t_{1}\right), \ldots, \tilde{u}\left(t_{k}\right)\right)$ is nondegenerate, because this by the implicit function theorem implies the contradiction that $\alpha_{1}$ is not the supremum. 
Thus, let us suppose by contradiction the intersection to be transversal and let us consider $\left(y_{1}, \ldots, y_{k}\right)$ such that

$$
D G^{\alpha_{1}}\left(\tilde{u}\left(t_{1}\right), \ldots, \tilde{u}\left(t_{k}\right)\right)\left(y_{1}, \ldots, y_{k}\right)=0 .
$$

We define

$$
h(t)=\left\{\begin{array}{c}
h_{0}(t), \quad t \leq t_{1} \\
h_{i}(t), \quad t_{i} \leq t \leq t_{i+1}, \quad 1 \leq i \leq k-1 \\
h_{k}(t), \quad t_{k} \leq t
\end{array}\right.
$$

where $h_{0}$ solves

$$
\left\{\begin{array}{c}
\ddot{h}=-V^{\prime \prime}(\tilde{u}, t) h-\alpha_{1} \dot{h} \\
h\left(t_{1}\right)=y_{1} \\
h(-\infty)=0
\end{array}\right.
$$

$h_{i}, 1 \leq i \leq k-1$, solves

$$
\left\{\begin{array}{c}
\ddot{h}=-V^{\prime \prime}(\tilde{u}, t) h-\alpha_{1} \dot{h} \\
h\left(t_{i}\right)=y_{i} \\
h\left(t_{i+1}\right)=y_{i+1}
\end{array}\right.
$$

and $h_{k}$ solves

$$
\left\{\begin{array}{c}
\ddot{h}=-V^{\prime \prime}(\tilde{u}, t) h-\alpha_{1} \dot{h} \\
h\left(t_{k}\right)=y_{k} \\
h(\infty)=0 .
\end{array}\right.
$$

The solutions of the first and the last problems exist since in $B_{p}(0, a) \times$ $B_{p}(0, a)$ the tangent spaces to the local stable and unstable manifolds of $\Phi_{\alpha_{1}}$ project surjectively on the configuration space. The solution to the second problem exists since we choose our partition $t_{1}<\ldots<t_{k}$ in such a way, that there are no Jacobi fields between $\tilde{u}\left(t_{i}\right)$ and $\tilde{u}\left(t_{i+1}\right)$. We have clearly that

$$
i_{\alpha_{1}}^{\prime}\left(\tilde{u}\left(t_{1}\right), \ldots, \tilde{u}\left(t_{k}\right)\right) \mathbf{y}=h
$$

where $i_{\alpha}$ is defined as in section 1 . We note that, since $\left(y_{1}, \ldots, y_{k}\right)$ is in the kernel of $D G^{\alpha_{1}}\left(\tilde{u}\left(t_{1}\right), \ldots, \tilde{u}\left(t_{k}\right)\right)$, then $h$ solves the linearized problem

$$
\left\{\begin{array}{c}
\ddot{h}=-V^{\prime \prime}(\tilde{u}, t) h-\alpha_{1} \dot{h} \\
h(-\infty)=0 \\
h(\infty)=0 .
\end{array}\right.
$$

But such a solution has the property that, for any $n \in \mathbf{Z},(h(n), \dot{h}(n))$ belongs to the intersection to the tangent spaces to the stable and unstable manifolds in $(\tilde{u}(n), \dot{\tilde{u}}(n))$. Since we are supposing that this intersection 
is transversal, we have that $\left(h\left(t_{1}\right), \dot{h}\left(t_{1}\right)\right)=0$ and thus that $h=0$. Thus, if $(\tilde{u}(n), \dot{\tilde{u}}(n))$ is a point of transversal intersection, we have that $\left(y_{1}, \ldots, y_{k}\right)=0$ and thus that $D G^{\alpha_{1}}\left(\tilde{u}\left(t_{1}\right), \ldots, \tilde{u}\left(t_{k}\right)\right)$ is nondegenerate, which we have seen to be absurd.

We are now going to show point ii) of the thesis.

We recall the hypotheses of ii) and the previous considerations imply that there is a sequence $\nu_{s} \searrow 0$ such that

$$
G^{\alpha_{1}-\nu_{s}}(\mathbf{x})=0 \Rightarrow D G^{\alpha_{1}-\nu_{s}}(\mathbf{x}) \text { is nondegenerate. }
$$

We note that $\nu_{s}>0 \forall s$, otherwise by the implicit function theorem $\alpha_{1}$ would not be the supremum. We suppose $\alpha_{1} \in\left(\gamma_{i}, \gamma_{i+1}\right]$. We take $s$ so big that $\alpha_{1}-\nu_{s}>\gamma_{i}, \alpha_{1}+\nu_{s}<\gamma_{i+2}$; we also consider $\bar{\Theta}$, the connected component of $\Gamma \cap \mathbf{R}^{k p} \times\left[\alpha_{1}-\nu_{s}, \alpha_{1}+\nu_{s}\right]$ containing $\left(\tilde{u}, \alpha_{1}\right)$. It follows from the definition of $\tilde{\Gamma}$ that $\bar{\Theta} \subset \tilde{\Gamma}$. From now on, we will consider $\nu_{s}$ fixed in this way. Since $\bar{\Theta}$ is a closed set of the compact $\tilde{\Gamma}$, it is compact and thus by (2.6) we have that $\bar{\Theta} \cap \mathbf{R}^{k p} \times\left\{\alpha_{1}-\nu_{s}\right\}$ consists of a finite number of nondegenerate points, say $\mathbf{y}_{1}, \ldots, \mathbf{y}_{r}$. We thus have that, for $\gamma \in(0, \delta]$ small enough,

$$
G^{\alpha_{1}-\nu_{s}}(\mathbf{x})=0, \quad\left(\mathbf{x}, \alpha_{1}-\nu_{s}\right) \in V_{\gamma}(\bar{\Theta}) \Rightarrow \mathbf{x} \in \bar{\Theta} .
$$

We now recall that, by our hypotheses, the zeroes of $G^{\alpha_{1}-\nu_{s}}$ correspond to transversal homoclinic intersections for $\Phi_{\alpha_{1}-\nu_{s}}$. Now Smale's homoclinic theorem (see for instance [12]) implies that, for $\varepsilon$ small enough and $m$ big enough, for any $l \geq 0$ and any choice of $i_{1}, \ldots, i_{2^{l}}$ there is one and only one solution $u$ of $\left(R^{\alpha}\right)$ such that, for any $j$,

$$
\left(u\left(t_{k}+j m\right), \dot{u}\left(t_{k}+j m\right)\right) \in B_{2 p}\left(\left(\mathbf{y}_{i_{j}}\right)_{k}, v_{\infty}^{\mathrm{dep}}\left(\left(\mathbf{y}_{i_{j}}\right)_{k}, \alpha_{1}-\nu_{s}\right), \varepsilon\right)
$$

where $\left(\mathbf{y}_{i_{j}}\right)_{k}$ denotes the coordinates of $\mathbf{y}_{i_{j}}$ which go from the $(k-1) p+1$ to the $k p$.

We note that, since $\bar{\Theta} \subset \tilde{\Gamma}$, then by definition of $\alpha_{1}, \bar{\Theta}$ does not intersect $\mathbf{R}^{k p} \times\{\alpha\}$ for $\alpha>\alpha_{1}$. Thus by the usual topological methods (see for instance [13], lemma 1.33, or [3]) we can find a compact set $K \supset \bar{\Theta}$, $K \subset \mathbf{R}^{k p} \times\left[\alpha_{1}-\nu_{s}, \alpha_{1}+\nu_{s}\right]$ and $\tilde{\delta} \in(0, \min (\gamma, \varepsilon)]$ such that

$$
V_{\tilde{\delta}}(K) \subset V_{\gamma}(\bar{\Theta}) \cap \mathbf{R}^{k p} \times\left(-\infty, \alpha_{1}+\nu_{s}\right)
$$

and

$$
\begin{aligned}
& \inf \left\{\left\|G^{\alpha}(\mathbf{x})\right\|_{\mathbf{R}^{k p}}:\right. \\
& \left.\quad(\mathbf{x}, \alpha) \in \partial V_{\tilde{\delta}}(K) \cap \mathbf{R}^{k p} \times\left[\alpha_{1}-\nu_{s}, \infty\right)\right\}=\chi>0 .
\end{aligned}
$$


Since, by (2.6) and the implicit function theorem, in a neighbourhood of each $\left(\mathbf{y}_{i}, \alpha_{1}-\nu_{s}\right), \bar{\Theta}$ is a smooth curve transversal to $\mathbf{R}^{k p} \times\left\{\alpha_{1}-\nu_{s}\right\}$, it follows easily that $K$ and $\tilde{\delta}$ can be chosen so that $V_{\tilde{\delta}}(K) \cap \mathbf{R}^{k p} \times\left\{\alpha_{1}-\nu_{s}\right\}$ has $r$ connected components, $E_{1}, \ldots, E_{r}$, each containing a point $\mathbf{y}_{i}$, and no other zero of $G^{\alpha_{1}-\nu_{s}}$. Since by (2.6) the zeroes of $G^{\alpha_{1}-\nu_{s}}$ are nondegenerate, by (2.7) we have that

$$
\forall i \in(1, \ldots, r), \quad \operatorname{deg}\left(G^{\alpha_{1}-\nu_{s}}, E_{i}, 0\right)= \pm 1
$$

From (2.4) we get that

$$
\left.\begin{array}{l}
\exists N \in \mathbf{N}: \quad \forall(\mathbf{x}, \alpha),(\mathbf{y}, \alpha) \in V_{\tilde{\delta}}(K), \quad \forall m \geq N \\
\quad\left|v_{m}^{\text {dep }}\left(x_{k}, y_{1}, \alpha\right)-v_{\infty}^{\text {dep }}\left(x_{k}, \alpha\right)\right| \\
\text { set } \quad+\left|v_{m}^{\text {arr }}\left(x_{k}, y_{1}, \alpha\right)-v_{-\infty}^{\text {arr }}\left(y_{1}, \alpha\right)\right|<\frac{\chi}{8} .
\end{array}\right\}
$$

We set

$$
C=V_{\tilde{\delta}}(K) \cap \mathbf{R}^{k p} \times\left[\alpha_{1}-\nu_{s}, \infty\right) .
$$

For $\alpha \in\left[\alpha_{1}-\nu_{s}, \alpha_{1}+\nu_{s}\right]$ and $m \geq N$ we define the operator

$$
\tilde{G}_{l}^{\alpha}: \prod_{j=1}^{2^{l}}\left(C \cap \mathbf{R}^{k p} \times\{\alpha\}\right) \subset \mathbf{R}^{2^{l} k p} \rightarrow \mathbf{R}^{2^{l} k p}
$$

by

$$
\begin{aligned}
\tilde{G}_{l}^{\alpha}\left(\mathbf{x}^{1}, \ldots, \mathbf{x}^{2^{l}}\right)= & \left(v^{\mathrm{dep}}\left(x_{1}^{1}, x_{2}^{1}, \alpha, 1\right)\right. \\
& -v_{m}^{\text {arr }}\left(x_{k}^{2^{l}}, x_{1}^{1}, \alpha\right), v^{\text {dep }}\left(x_{2}^{1}, x_{3}^{1}, \alpha, 2\right) \\
& -v^{\text {arr }}\left(x_{1}^{1}, x_{2}^{1}, \alpha, 2\right), \ldots, v_{m}^{\text {dep }}\left(x_{k}^{1}, x_{1}^{2}, \alpha\right) \\
& -v^{\text {arr }}\left(x_{k-1}^{1}, x_{k}^{1}, \alpha, k\right), v^{\text {dep }}\left(x_{1}^{2}, x_{2}^{2}, \alpha, 1\right) \\
& -v_{m}^{\text {arr }}\left(x_{k}^{1}, x_{1}^{2}, \alpha\right), v^{\text {dep }}\left(x_{2}^{2}, x_{3}^{2}, \alpha, 2\right) \\
& -v^{\text {arr }}\left(x_{1}^{2}, x_{2}^{2}, \alpha, 2\right), \ldots, v_{m}^{\text {dep }}\left(x_{k}^{2^{l}}, x_{1}^{1}, \alpha\right) \\
& \left.-v^{\text {arr }}\left(x_{k-1}^{2^{l}}, x_{k}^{2^{l}}, \alpha, k\right)\right)
\end{aligned}
$$

As is section 1, it is possible to show that the zeroes of $\tilde{G}_{l}^{\alpha}$ correspond to solutions of $\left(R^{\alpha}\right)$ of period $2^{l}\left(m+t_{k}-t_{1}\right)$. Formula (2.13) can also be written as

$$
\begin{aligned}
& \tilde{G}_{l}^{\alpha}\left(\mathbf{x}^{1}, \ldots, \mathbf{x}^{2^{l}}\right)=\left(G^{\alpha}\left(\mathbf{x}^{1}\right), \ldots, G^{\alpha}\left(\mathbf{x}^{2^{l}}\right)\right) \\
& \quad+\left(v_{-\infty}^{\operatorname{arr}}\left(x_{1}^{1}, \alpha\right)-v_{m}^{\operatorname{arr}}\left(x_{k}^{2^{l}}, x_{1}^{1}, \alpha\right), 0, \ldots, 0, v_{m}^{\operatorname{dep}}\left(x_{k}^{1}, x_{1}^{2}, \alpha\right)\right. \\
& \left.\quad-v_{\infty}^{\operatorname{dep}}\left(x_{k}^{1}, \alpha\right), v_{-\infty}^{\operatorname{arr}}\left(x_{1}^{2}, \alpha\right)-v_{m}^{\operatorname{arr}}\left(x_{k}^{1}, x_{1}^{2}, \alpha\right), 0, \ldots\right)
\end{aligned}
$$


We set

$$
A_{l}=\bigcup_{\alpha \in\left[\alpha_{1}-\nu_{s},+\infty\right)} \prod_{j=1}^{2^{l}}\left(C \cap \mathbf{R}^{k p} \times\{\alpha\}\right)
$$

We note that, by (2.9) and the definition of $C$,

$$
A_{l} \subset \mathbf{R}^{2^{l} k p} \times\left[\alpha_{1}-\nu_{s}, \alpha_{1}+\nu_{s}\right) .
$$

It is easy to see that $A_{l}$ is an open set in $\mathbf{R}^{2^{l} k p} \times\left[\alpha_{1}-\nu_{s}, \alpha_{1}+\nu_{s}\right]$. The same argument which yielded (1.17) now yields that by (2.10) and (2.12) we have

$$
\begin{aligned}
& \inf \left\{\left\|\tilde{G}_{l}^{\alpha}\left(\mathbf{x}^{1}, \ldots, \mathbf{x}^{2^{l}}\right)\right\|_{\mathbf{R}^{2^{l} k p}}:\right. \\
& \left.\quad l \in \mathbf{N} \cup\{0\},\left(\mathbf{x}^{1}, \ldots, \mathbf{x}^{2^{l}}, \alpha\right) \in \partial A_{l} \cap \mathbf{R}^{2^{l} k p} \times\left[\alpha_{1}-\nu_{s}, \infty\right)\right\} \geq \frac{\chi}{2} .
\end{aligned}
$$

Moreover, (2.10), (2.11), (2.12) and (2.14) imply, by the same arguments that yielded (1.18), that, if $D_{l}$ is a connected component of $A_{l} \cap \mathbf{R}^{2^{l} k p} \times$ $\left\{\alpha_{1}-\nu_{s}\right\}$, then

$$
\forall l \in \mathbf{N} \cup\{0\}, \quad \operatorname{deg}\left(\tilde{G}_{l+1}^{\alpha_{1}-\nu_{s}}, D_{l} \times D_{l}, 0\right)=1
$$

and that, for any $i_{1}, \ldots, i_{2^{t}} \in(1, \ldots, r)$,

$$
\operatorname{deg}\left(\tilde{G}_{l}^{\alpha_{1}-\nu_{s}}, E_{i_{1}} \times \ldots \times E_{i_{2^{l}}}, 0\right)= \pm 1 \text {. }
$$

By (2.8) and our choice of $\tilde{\delta}$ it follows that $E_{i_{1}} \times \ldots \times E_{i_{2} l}$ contains only one zero of $\tilde{G}^{\alpha_{1}-\nu_{s}}$.

We now note that

$$
\tilde{G}_{l}^{\alpha}(\mathbf{x})=0 \Rightarrow \tilde{G}_{l+1}^{\alpha}(\mathbf{x}, \mathbf{x})=0
$$

and that $(\mathbf{x}, \mathbf{x})$ corresponds to a solution of $\left(R^{\alpha}\right)$ of period $2^{l+1}\left(m+t_{k}-t_{1}\right)$ which is also a solution of $\left(R^{\alpha}\right)$ of period $2^{l}\left(m+t_{k}-t_{1}\right)$. We set $\bar{\Theta}_{0}=\bar{\Theta}$ and define $\bar{\Theta}_{l}$ inductively as the maximal continuum of zeroes of $G_{l}^{\alpha}$ in the closure of $A_{l}$ containing

$$
\bar{\Theta}_{l-1}^{2}=\bigcup_{\alpha \in\left[\alpha_{1}-\nu_{s}, \alpha_{1}+\nu_{s}\right]}\left\{(\mathbf{x}, \mathbf{x}, \alpha):(\mathbf{x}, \alpha) \in \bar{\Theta}_{l-1}\right\} .
$$

Clearly, we have by (2.16) that

$$
\bar{\Theta}_{l} \subset A_{l}, \quad \forall l .
$$


it is clear that point (ii) of the thesis amounts to showing that, for $l \geq 1$, $\bar{\Theta}_{l+1}$ properly contains $\bar{\Theta}_{l}^{2}$. Thus let us suppose by contradiction that, for some $l, \bar{\Theta}_{l+1}=\bar{\Theta}_{l}^{2}$. By the usual topological methods we can find a neighbourhood $W$ of $\bar{\Theta}_{l}^{2}$ such that

$W \subset A_{l+1}$,
$\inf \left\{\left\|G_{l}^{\alpha}\left(\mathbf{x}_{1}, \ldots, \mathbf{x}_{2^{l+1}}\right)\right\|_{\mathbf{R}^{2^{l+1} k_{p}}}:\left(\mathbf{x}_{1}, \ldots, \mathbf{x}_{2^{l+1}}\right) \in \partial W\right\}>0$.

We now note that $\bar{\Theta}_{l}^{2} \cap \mathbf{R}^{2^{l+1} k p} \times\left\{\alpha_{1}-\nu_{s}\right\}$ is contained in $\bigcup_{j} D_{j} \times D_{j}$, where $D_{j}$ is a connected component of $A_{l} \cap \mathbf{R}^{2^{l} k p} \times\left\{\alpha_{1}-\nu_{s}\right\}$. We have then that $D_{j}=E_{i_{1}} \times \ldots \times E_{i_{2} l}$. But, by our previous considerations, in $D_{j} \times D_{j}$ there is at most one zero of $\tilde{G}_{l+1}^{\alpha_{1}-\nu_{s}}$, which thus must be an element of $\bar{\Theta}_{l}^{2}$. Thus by (2.17), nondegeneracy and the excision formula for the degree we have that

$$
\bar{\Theta}_{l}^{2} \cap D_{j} \times D_{j} \neq \varnothing \Rightarrow \operatorname{deg}\left(\tilde{G}_{l+1}^{\alpha_{1}-\nu_{s}}, W \cap\left(D_{j} \times D_{j}\right), 0\right)=1
$$

which, since $\bar{\Theta}_{l}^{2} \cap \mathbf{R}^{2^{l+1} k p} \times\left\{\alpha_{1}-\nu_{s}\right\}$ is contained in $\bigcup_{j} D_{j} \times D_{j}$, implies that

$$
\operatorname{deg}\left(\tilde{G}_{l+1}^{\alpha_{1}-\nu_{s}}, W \cap \mathbf{R}^{2^{l+1} k p} \times\left\{\alpha_{1}-\nu_{s}\right\}, 0\right)>0 .
$$

The last formula now implies that $\bar{\Theta}_{l}^{2}$ can be continued above $\alpha_{1}-\nu_{s}$, which is in contradiction with (2.15) and (2.16).

\section{REFERENCES}

[1] K. Alligood and J. Yorke, Cascades of Period-doublig Bifurcations: a Prerequisite for Horseshoes, Bull. Amer. Math. Soc., Vol. 9, 1983, pp. 319-323.

[2] U. Bessi, A Variational Proof of a Sitnikov-like Theorem, to appear on Nonlin. Anal., T.M.A.

[3] U. BESSI, Global Homoclinic Bifurcation for Damped Systems, to appear on Math. Zeit.

[4] S. N. Chow, J. K. Hale and J. Mallet-Paret, An Example of Bifurcation to Homoclinic Orbits, J.D.E., Vol. 37, 1980, pp. 351-373.

[5] V. Coti Zelati, I. Ekeland and E. SÉRE, A Variational Approach to Homoclinic Orbits in Hamiltonian Systems, Math. Ann., Vol. 288, 1990, pp. 133-160.

[6] V. Coti Zelati and P. H. Rabinowttz, Homoclinic Orbits for Second Order Hamiltonian Systems Possessing Superquadratic Potentials, J. Amer. Math. Soc., Vol. 4, I991, pp. 693-727.

[7] J. Franks, Period Doubling and the Lefschetz Formula, Trans. Am. Math. Soc., Vol. 1, 1985, pp. 275-283.

[8] J. LeRAy and J. Schauder, Topologie et Équations fonctionnelles, Ann. Scien. École norm. Sup., 1934, pp. 45-78. 
[9] S. MATHLoutHI, Bifurcation d'orbites homoclines pour les systèmes hamiltoniens, Annales de la Faculté des Sciences de Toulouse, Vol. 1, 1992, pp. 211-236.

[10] A. Marino and G. Prodi, Metodi Perturbativi nella Teoria di Morse, Boll. U.M.I., Vol. 11, 1975 , pp. 1-32.

[11] M. Misiurewitz, On Non-continuity of Topological Entropy, Bull. Acad. Pol. Sci., Vol. 19, 1971, pp. 319-320.

[12] S. NewhousE, Lectures on Dynamical Systems, C.I.M.E. Summer School at Bressanone, Italy, Birkhaueser, Boston, 1980.

[13] P. H. Rabinowitz, Nonlinear Sturm-Liouville Problems for Second Order O.D.E., Comm. Pure and Appl. Math., Vol. 23, 1970, pp. 939-961.

[14] E. SÉRÉ, Looking for the Bernoulli Shift, Ann. Inst. H. Poincaré, Anal. non Linéaire, Vol. 10, 1993, pp. 561-590.

(Manuscript received November 3, 1993;

revised March 2, 1994.) 